\title{
New survey medium: Collecting marketing data with e-mail and the World Wide Web
}

Received: 21st November, 2000

\section{Sally Dibb}

is a reader in Marketing and Strategic Management at Warwick Business School where she is also Associate Dean (Undergraduate Programmes). She lectures on marketing analysis, strategic marketing and buyer behaviour.

\section{Anna Rushmer}

gained an MSc in Operations Research at Warwick Business School in 1999 before joining Fair Isaac in Birmingham.

\section{Philip Stern}

is a lecturer in Marketing and Strategic Management at Warwick Business School. His research focuses on market segmentation, the pharmaceutical industry and the prescribing behaviour of general practitioners.

\section{Sally Dibb}

Warwick Business School, University of Warwick, Coventry CV4 7AL, UK.

Tel: +44 1203 522801; Fax: +44 1203 524628; e-mail: MSMSD@wbs. warwick.ac.uk

\section{INTRODUCTION}

This paper describes the use of e-mail as a survey medium for collecting data from marketing academics. Although at an early stage of development, the literature suggests that this data collection approach may offer a cheap, quick and effective means for collecting information. Not surprisingly, researchers from a range of disciplines are now considering data collection via this means. The application of e-mail as a survey medium remains relatively untested however. This paper helps to bridge the knowledge gap by testing the effectiveness of data collection by e-mail. As the key objective of this research was to test a particular survey medium, the topic area of the questionnaire was incidental. It was necessary, however, to select subject matter that was sufficiently conceptually taxing to allow the medium to be tested rigorously. For this reason, the questionnaire sought academic views on market segmentation, a complex and widely researched area of the marketing theory.

The specific objectives of the paper are to examine the effectiveness of e-mail as a survey medium by carrying out a data collection experiment. This allows published assumptions about the appropriateness of an e-mail approach to be tested, so that recommendations can be made to assist researchers adopting the method. The paper begins by reviewing 
the literature on the advantages and disadvantages of e-mail and the Internet as survey media. It is important to emphasise that the paper focuses on the effectiveness of the data collection approach and that the findings of the segmentation questionnaire are incidental. The implications from the data analysis are presented together with a series of recommendations for those wishing to use an e-mail survey approach.

\section{SELECTING A SURVEY MEDIUM}

Most marketing academics are familiar with the relative advantages and disadvantages of different survey media. A detailed account of these can be found in many marketing and statistical texts. ${ }^{1}$ When selecting a particular medium the researcher will carefully weigh up the advantages and disadvantages of the different approaches before making an informed choice about how to proceed. For many researchers, issues of cost and response speed are crucial when making such decisions. ${ }^{2}$ In such cases, certain new technology approaches may seem particularly attractive. For instance, the popularity of the fax machine as a data collection tool is partly linked to the need for fast distribution at low cost. ${ }^{3}$

The idea of using e-mail as an alternative to conventional survey media was introduced by Sproull. ${ }^{4}$ Although few research studies have been conducted using e-mail, the wide-ranging advantages of this method suggest a bright future. The advantages/ disadvantages of the use of e-mail compared with the more traditional mail survey are reviewed below. Further details can be found in Kiesler et al., ${ }^{5}$ Mehta and Sivadas, ${ }^{6}$ Parker, ${ }^{7}$ Resnick ${ }^{8}$ and Tse. ${ }^{9}$

The advantages of e-mail as a survey medium are:
- low transmission and collection costs. This is particularly attractive for international data collection

- convenience of handling. E-mail files can be saved into the outgoing mailbox and sent on to a large number of addresses simply by entering the respondent's address. This reduces the physical effort associated with collating questionnaires, stuffing and addressing envelopes

- the sender is immediately notified of incorrect addresses

- responses are returned with a notification of time and date

- respondent queries about completing the questionnaire can be handled quickly

- e-mail respondents tend to be less inhibited in their answers, writing more for open-ended questions

- transmission is very fast. E-mail can be sent anywhere in the world almost instantaneously

- a better level of sample control may be achieved than for mail-based surveys. Although it is common for colleagues or secretaries to open another colleague's mail, most individuals deal with their own e-mail

- e-mail questionnaires are currently relatively unusual and may therefore arouse respondents' interest and attention

- more urgency may be attached to an e-mail message and it is less likely to be perceived as junk mail than a mailed questionnaire

- e-mails are perceived as an environmentally-friendly means of communication.

The disadvantages of e-mail as a survey medium are:

— in many circumstances, sample coverage is low. Distribution is restricted to those with access to 
e-mail. In addition, those targeted need to be sufficiently confident to use this medium

- sample bias may arise from the low sample coverage. Research suggests that those most likely to answer an e-mail survey are likely to be well educated, upper/middle income individuals interested in technology

- the quality and appearance of an e-mail questionnaire may not be as good as a mailed questionnaire

- it is more difficult to include tangible incentives. This may lead to a reduced response rate

- not everyone checks e-mail on a daily basis and so, unless the date of reading the e-mail is checked against the questionnaire return date, the average response speed may be underestimated

- respondents who receive a large quantity of e-mail may respond negatively to being surveyed by this route and may discard the e-mail as junk mail. Some respondents may resent receiving unsolicited mail

- some respondents may find an e-mail questionnaire more difficult to respond to than one received by mail

- e-mail makes it difficult for respondents to remain anonymous. This may affect willingness to respond.

Common themes emerging from the literature on survey media include survey costs, response rates, speed of return and quality of responses. On the question of survey costs researchers generally agree that sending e-mail questionnaires is extremely inexpensive when compared to other media such as mail or interviews. ${ }^{10}$ $\mathrm{Tse}^{11}$ and Mehta and Sivadas ${ }^{12}$ suggest an average minimum cost of $\$ 0.54$ for a mail survey. By comparison, there are no direct costs involved with sending e-mails. There is also widespread agreement about the fast response associated with e-mail surveys. Sproull's research shows that average return times for electronic questionnaires are half that achieved by conventional returns. Similar findings are shown by Tse who indicated an average e-mail response time of 142 hours (just under six days) ahead of those returned by mail. Mehta and Sivadas report even more favourable response speeds, with over 50 per cent of e-mail questionnaires returned within two to three days, compared with three weeks taken to receive the same number by mail. Researchers are also positive about response quality for e-mail surveys, suggesting that there is little difference in comparison to similar mail questionnaires. While Mehta and Sivadas found comparable completeness levels for both media, it is possible, however, that e-mail respondents may be willing to provide more detail in open-format questions.

Opinions about overall response rates for e-mail surveys are less consistent. In a worldwide study, Mehta and Sivadas suggest that the response rate for an e-mail questionnaire sent with pre-notification is only slightly lower than a mailed questionnaire sent with pre- and post-notifications and offering an incentive. Tse's research, carried out in Hong Kong, is less encouraging. Only 7 per cent of those targeted by e-mail responded, while 52 per cent of those mailed returned their questionnaire. In this particular case, the low e-mail response rate can be partially explained by the fact that e-mail was a relatively new technology in Hong Kong at the time. This caused Tse to propose a series of conditions under which e-mail surveys may be conducted. First, the population under study must have very high e-mail account ownership. Secondly, the inclusion of incentives must be unlikely to increase substantially response rate and 
quality. Thirdly, it should be unnecessary to use high quality image or colour within the questionnaire. As the discussion later in the paper will show, these guidelines remain an important consideration for researchers seeking to use electronic data collection approaches.

In summary, the literature identifies a range of potential advantages for using e-mail as a survey medium. Relatively low cost and convenience are among the most important. To capitalise on these advantages certain conditions relating to the appropriateness of e-mail for the sample population must be satisfied. It is also important to consider that some respondents remain cautious about returning questionnaires through this route and that this may impact upon the quality/rate of response.

\section{DATA COLLECTION}

The data collection tool of choice was a questionnaire hosted on the World Wide Web. Potential respondents received a URL address in the form of a hypertext link contained within an e-mail message. The e-mail invited respondents to visit the web page to complete the questionnaire. The attractions of a combined e-mail and Web approach are similar to those already indicated. Thus Schillewaert et al. agree that Web-based surveys are cost effective, convenient and flexible, conditions necessary for this particular research. ${ }^{13}$ In this particular case, using e-mail also allowed the researcher to exert a high level of control over the sample so that it could be closely determined and monitored.

As the literature review indicates, there are various circumstances in which this survey medium is an appropriate one to choose. In this research, the reasons were clear cut. First, the population under study consisted of individuals who would, in almost all cases, have an e-mail address. The prevalence of e-mail within the university sector is widely recognised, and most university academics are familiar with this form of communication. Secondly, the popularity of e-mail as a medium meant that it was highly likely that those surveyed would regularly check their account for incoming mail and would receive the questionnaire quickly. As e-mail and the World Wide Web are the same type of medium, those interested in the research would probably find it convenient to visit the web page immediately. This would have a positive impact upon the speed and size of response.

By combining e-mail and the World Wide Web it was possible to benefit from the low costs and fast response rate associated with e-mail, while retaining a greater degree of control over the questionnaire layout. It is notoriously difficult to control the appearance of a research instrument delivered by e-mail. Any disruption to the layout of a questionnaire can cause problems for respondents and may affect data quality. ${ }^{14}$ Using a web page these difficulties can be avoided and the visual appearance/layout assured.

When creating web pages, users are faced with a number of options, principally writing hypertext mark-up language (HTML) directly into an editor or using specialist software packages. Writing HTML directly produces more efficient code which is easier to maintain. It is also, however, much less flexible and more difficult for the novice or occasional web page designer to use. Given that the leading web browsers include user-friendly software specifically developed for web page design, it was decided to use Netscape's 'Composer' to create the web-based questionnaire. The key steps are described below:

— the final questionnaire draft was 
converted from a 'Word' document to HTML format. This allowed the file to be opened by the standard browser tools eg Netscape.

- a number of formatting changes were undertaken using features of Composer. This primarily involved simple modifications to the layout of tables. In one case, the structure of a table was altered because it appeared too confusing for electronic transmission.

- a number of HTML tags were added to the web page. Tags are objects which are added either to allow the user greater interaction or to introduce the possibility of two-way communication. Tags can take the form of radio buttons, check boxes or text boxes. They allow the respondent to enter information by selecting buttons or entering text into boxes. These are the building blocks for designing a web-based survey instrument. Four different tag types were used for creating the web page: radio buttons (used when only one from a number of answers is to be selected); check boxes (appropriate when more than one answer may be required for a question); text entry fields (permit the inputting of a small amount of text or numerical data); text area tags (applicable for open-ended questions when more sizeable amounts of text are required) and spacer tags (control the horizontal spacing of elements of the web page)

- a hypertext link was set up to allow the respondent to contact the researcher if any clarification of the research instrument was required

- decisions were made about the appropriate colour for the questionnaire. A light pastel yellow background was selected with bright red text. This provided contrast with the blue colour of the e-mail contact address. Different colours were used for alternate rows of the tabular questions to assist reading

- the web page was published with an appropriate introductory statement thanking respondents for visiting the site. Instructions were also provided on how to submit and reset the form and contact e-mail address. The publication process involved copying the HTML file onto a home directory, then changing UNIX permissions so that the file could be publicly read.

The subject matter for the questionnaire was academic views on market segmentation, with respondents questioned about the following areas:

— value of segmentation in improving customer understanding and enhancing organisational performance

- effectiveness of various segmentation techniques (eg cluster analysis, MDS etc.) in helping to a) understand the customer and b) improve organisational performance

- usefulness of various methods for evaluating segmentation results

- current/changing role of segmentation in the marketing curriculum/research agenda.

In view of the uncertainties associated with the delivery and appearance of the research instrument, careful testing was crucial. Following publication of the web page a two-stage on-line test of the questionnaire was conducted. The first stage involved seeking peer evaluation from a small number of locally-based marketing academics. Three individuals provided feedback on the format and content of the questionnaire. This feedback was actioned, with appropriate modifications to the wording and layout of the research instrument. The second 
stage test comprised a formal pilot. The e-mail addresses of 166 marketing academics who were members of the European Marketing Academy (EMAC) were selected from the membership directory. Members from the following countries were included in the pilot: Belgium, Denmark, Ireland, New Zealand, Spain and the USA. This represented one third of the planned sample size for the main data collection phase. The first five of these countries were chosen because they were not to be included in the main data collection. The pilot survey response rate was 22 per cent.

The e-mail invitation to participate in the research was then sent to a sample of 484 EMAC members from Australia, France, Germany, Sweden and the UK. A number of US universities were also selected to represent institutions whose marketing faculty would classify themselves as research active. Individual e-mail addresses were collected by visiting the relevant institutions' websites. None of the US academics contacted for the pilot study were targeted for this part of the research. E-mails were sent midweek, in the afternoon, to allow response times to be recorded and to minimise the problems created by the weekend break. A cut-off point of one week was allowed for responses. Once received, the responses were pasted into a Microsoft Excel workbook for analysis. Out of the 484 questionnaires sent, 46 were returned as undeliverable. Of the 438 remaining, 96 were filled, representing a 22 per cent response rate.

\section{RESULTS}

The purpose of this paper is to consider the effectiveness of using a combined e-mail and Web data collection approach and to make appropriate recommendations. Reviewing the market segmentation data elicited from the questionnaire is therefore outside the scope of this paper. Those interested in learning more about the market segmentation data should see Rushmer. ${ }^{15}$

The effectiveness of the e-mail/Web data collection can be appraised on the basis of survey costs, overall response rate, speed of response and quality of responses. First point, the financial costs of administering the survey were negligible. Although it took some days to set up the questionnaire on the Web, the time involved to collect data through another route (eg mail or telephone) would have been much greater. Moving on to response rate, timing and time constraints meant that the 22 per cent level achieved was acceptable. Due to researcher availability the survey took place in August, which for many academics falls within the summer vacation. This inevitably reduced the response rate. Time constraints requiring a cut-off return date of one week after the questionnaire was distributed were also likely to curtail the response rate. Of course, such constraints would have completely ruled out certain other data collection media.

The 22 per cent response rate is almost identical to that reported by Dommeyer and Moriarty who surveyed students by attached and embedded e-mail instruments, but lower than other studies they reference. ${ }^{16}$ Of course it is important to consider that prior studies are not directly comparable because they have only considered e-mail, and not as here an e-mail/Web linked approach. This merely reinforces the relatively innovative nature of this kind of data collection and the need for tests of this kind.

Speed of response to the questionnaire was extremely fast. Forty-four per cent of responses were received on the same day as transmission. This is particularly 
impressive considering that response speed was calculated using British

Standard Time. Thus questionnaires were all distributed at the same point in time.

The implication is that in some countries (eg France) the recipients were reaching the end of their working day, while in others (eg the USA), they were at the beginning. A further 31 per cent of responses were returned on the following day. After this, there was a dramatic reduction in returns with 9 per cent, 3 per cent, 1 per cent, 9 per cent and 2 per cent being received on each of the next five days respectively. This supports the notion that respondents choosing to complete a questionnaire distributed via e-mail will do so upon reading. Note that the sudden reduction in return rates on days four and five ( 3 per cent and 1 per cent respectively) coincided with the weekend. This also explains the increase in responses to 9 per cent on day six (the following Monday). Overall, these findings would apparently indicate that e-mail is a particularly attractive medium when time is a major constraint.

The data quality can be assessed by reviewing the completeness of questionnaire responses. In order to provide a rigorous test, the questionnaire included a mix of simple tick-box and more complex scaled questions in combination with open format questions. For the closed format questions the figures for non-response were very low with between 0 and 10 per cent of answers missing for each question. As would be expected the level of non-response was much higher for the open format questions, ranging from 23 per cent in the early questions to 62 per cent towards the end of the questionnaire. Where open-ended questions were completed, responses were detailed and insightful, often running to several sentences, suggesting that respondents were not adversely affected by the medium. It is clear, however, that the medium can also cause problems with data quality. One particularly complex tabular question was not transmitted cleanly on certain browsers, leading to some cases of non-response. This emphasises the importance of being able to control the appearance of the survey medium, irrespective of where it is sent.

\section{CONCLUSIONS AND IMPLICATIONS}

On the basis of cost, response rates, speed of response and data quality the survey medium test can be deemed a success. The findings support the assertions in the literature about the low costs associated with e-mail surveys. Response rates and data quality were within a reasonable range and response speeds were excellent. Taking into account the problems associated with the research timing, this reaffirms the appropriateness of the medium for this particular respondent group. It is equally clear, however, that researchers seeking to use electronic means of data collection must carefully appraise its suitability. At this stage it is therefore helpful to revisit Tse's conditions under which e-mail surveys may be conducted. ${ }^{17}$

First, as Tse indicated, a high proportion of e-mail account ownership is essential. In this respect, the high level of e-mail usage among academics substantially enhanced the feasibility of the research. Tse's second concern related to the problems of sending research incentives via e-mail. The implication is that an electronic approach to data collection may be unsuitable in circumstances where an incentive is needed to ensure high response rates. For this particular research, the inclusion of an incentive was unnecessary, so the problem did not arise. In circumstances 
requiring inducement, however, researchers should consider a range of on-line incentives. For example, respondents might receive Internet currency, free software or free access to certain websites. Alternatively, competition entry could be offered, with the administrative details handled by e-mail and prizes being mailed by traditional means. Tse's final concern relates to the difficulty in incorporating high quality images or colour within the questionnaire. It is certainly true that researchers who use a simple e-mail approach have relatively little control over how the research instrument appears on a recipient's machine. By using a combined e-mail and World Wide Web approach, however, disruption to the required visual appearance and layout can, as in this case, be avoided. On the basis of this assessment, it seems that the only substantive barrier to electronic data collection is whether or not potential respondents have e-mail/WWW access and are familiar with its use.

For those deciding to collect their data by e-mail, this research signals a number of guidelines which can greatly assist implementation:

— timing of the e-mail: if accurate response speeds are required for an international study, all countries must receive the questionnaire at the same local time. Some e-mail subscribers disable confirmation of receipt and reading software so this option may not obviate the requirement for a country-based batch approach in international studies. Weekends should also be avoided for questionnaires targeted at the workplace, but may be preferred for those targeted at consumers in their own homes

- on-screen appearance: this research found the on-screen appearance of the questionnaire at the point of response cannot be guaranteed in the way that a mailed questionnaire can. Using a web page helps considerably, but more comprehensive testing of the questionnaire using a variety of different browsers may be appropriate

- identifying respondents: it is preferable to tag respondents with some kind of identifier as, in theory, an individual can visit the website and answer the questionnaire on many occasions - expediting data input: the possibilities of data input errors can be reduced and time saved by linking respondent's replies directly to a spreadsheet or other data capture software.

It is inevitable that the considerable attractions of e-mail data collection will radically enhance its popularity. Although the application of e-mail/WWW data collection is limited to survey populations which are heavy e-mail/Internet users, growing penetration of these media suggests a promising future. Verdict research ${ }^{18}$ suggests that within five years around 55 per cent of UK households will own a PC, many of which will have e-mail accounts. Twenty-five per cent of households will have digital services (with e-mail facilities) via their televisions. Others will be able to access the Internet using Wireless Application Protocol (WAP) over their mobile telephones. ${ }^{19}$ As the use of this media grows, however, e-mail users will receive an increasing amount of unsolicited research material and the novelty of completing questionnaires via this route will sharply decline. This will have repercussions for response rates. Further research is needed which appraises the effectiveness of this medium on an ongoing basis and within a variety of contexts. For now though it seems that the time is ripe for seizing the 
opportunities on offer from this exciting survey medium.

\section{References}

1 Parasuraman, A. (1991) 'Marketing research', Addison Wesley, Reading, Massachusetts.

2 Kazuaki, K. (1990) 'Recent developments and future trends in marketing research in Japan using new electronic media', Journal of Advertising Research, Vol. 30, pp. 53-57.

3 Dickson, J. and MacLachlan, D. (1996) 'Fax surveys: Return patterns and comparison with mail surveys', Journal of Marketing Research, Vol. 33, No. 1, pp. 108.

4 Sproull, L. (1986) 'Using electronic mail for data collection in organizational research', Academy of Management Journal, Vol. 29, No. 1, pp. 159-169.

5 Kiesler, S., Siegler, J. and McGuire, T. (1984) 'Social psychological aspects of computer-mediated communication', American Psychologist, Vol. 39, pp. 1123-1234.

6 Mehta, R. and Sivadas, E. (1995) 'Comparing response rates and response content in mail versus electronic mail surveys', Journal of the Market Research Society, Vol. 37, No. 4, pp. 429-439.

7 Parker, L. (1992) 'Collecting data the e-mail way',
Training and Development, pp. 52-54.

8 Resnick, R. (1994) 'Hitching a ride into cyberspace', Nation's Business, pp. 66-68.

9 Tse, A. (1998) 'Comparing the response rate, response speed and response quality of two methods of sending questionnaires: e-mail vs. mail', Journal of the Market Research Society, Vol. 40, No. 4, pp. 353-363.

10 Sproull (1986) op. cit.

11 Tse (1998) op. cit.

12 Mehta and Sivadas (1995) op. cit.

13 Schillewart, N., Langerak, G. and Duhamel, T. (1998) 'Non-probability sampling for WWW surveys: A comparison of methods', Journal of the Market Research Society, Vol. 40, No.4, pp. 307-317.

14 Dommeyer, C.J. and Moriarty, E. (2000) 'Comparing two forms of an e-mail survey: Embedded vs. attached', International Journal of Marketing Research, Vol. 42, No. 1, pp. 39-50.

15 Rushmer, A. (1999) 'Academic views of market segmentation', Warwick MBA Project, Warwick Business School.

16 Dommeyer and Moriarty (2000) op. cit.

17 Tse (1998) op. cit.

18 Verdict (2000) 'Verdict on electronic shopping 2000', http://www.verdict.co.uk/es2000.htm.

19 Reed, M. (2000) 'Why the future will be wireless', Marketing, 3 February, pp. 22-23. 